\title{
Preparation and evaluation of an Arg-Gly-Asp-modified chitosan/hydroxyapatite scaffold for application in bone tissue engineering
}

\author{
LIN CHEN $^{1 *}$, BAOLIN LI $^{2 *}$, XIAO XIAO ${ }^{2}$, QINGGANG MENG $^{2}, \mathrm{WEI} \mathrm{LI}^{2}$, \\ QIAN YU ${ }^{3}$, JIAQI $\mathrm{BI}^{2}$, YONG $\mathrm{CHENG}^{2}$ and ZHIWEI QU ${ }^{2}$ \\ ${ }^{1}$ Department of Pathogenic Microorganisms, The Second Affiliated Hospital, Harbin Medical University, \\ Harbin, Heilongjiang 150081; ${ }^{2}$ Department of Orthopedic Surgery, The First Hospital of Harbin City, \\ Harbin Medical University, Harbin, Heilongjiang 150001; ${ }^{3}$ Department of Orthopedics, \\ The Fourth Affiliated Hospital, Harbin Medical University, Harbin, Heilongjiang 150081, P.R. China
}

Received November 12, 2014; Accepted August 7, 2015

DOI: $10.3892 / \mathrm{mmr} .2015 .4371$

\begin{abstract}
Bone tissue engineering has become a promising method for the repair of bone defects, and the production of a scaffold with high cell affinity and osseointegrative properties is crucial for successful bone substitute. Chitosan (CS)/hydroxyapatite (HA) composite was prepared by in situ compositing combined with lyophilization, and further modified by arginine-glycine-aspartic acid (RGD) via physical adsorption. In order to evaluate the cell adhesion rate, viability, morphology, and alkaline phosphatase (ALP) activity, the RGD-CS/HA scaffold was seeded with bone marrow stromal cells (BMSCs). The osseointegrative properties of the RGD-CS/HA scaffold were evaluated by in vivo heterotopic ossification and in vivo bone defect repair. After $4 \mathrm{~h}$ culture with the RGD-CS/HA scaffold, the adhesion rate of the BMSCs was $80.7 \%$. After 3 days, BMSCs were fusiform in shape and evenly distributed on the RGD-CS/HA scaffold. Formation of extracellular matrix and numerous cell-cell interactions were observed after $48 \mathrm{~h}$ of culture, with an ALP content of $0.006 \pm 0.0008 \mathrm{U} / \mathrm{l} / \mathrm{ng}$. Furthermore, the osseointegrative ability and biomechanical properties of the RGD-CS/HA scaffold were comparable to that of normal bone tissue. The biocompatibility, cytocompatibility, histocompatibility and osseointegrative properties of the RGD-CS/HA scaffold support its use in bone tissue engineering applications.
\end{abstract}

Correspondence to: Professor Zhiwei Qu, Department of Orthopaedic Surgery, The First Hospital of Harbin City, Harbin Medical University, 149 Mai Mai Street, Daoli, Harbin, Heilongjiang 150001, P.R. China

E-mail: quzhiwei2@163.com

Key words: bone tissue engineering, chitosan/hydroxyapatite scaffold, osseointegration, arginine-glycine-aspartic acid modification

\section{Introduction}

The treatment of bone defects arising from traumatic events, congenital abnormalities and infection involves autografting and allografting of bone tissues $(1,2)$, or replacement surgery (3-5). The satisfactory clinical outcomes of autografting and allografting (6) are tempered, however, by problems of insufficient supply, significant morbidity and risk of disease transmission, promoting the development of alternative technologies, such as bone tissue engineering $(7,8)$. The primary purpose of bone tissue engineering is to generate synthetic bone grafting substitutes through the combination of scaffolding material, viable cells and growth factors. In particular, biocompatible scaffolds are garnering increased attention due to their provision not only of structural support for cells, but also of an environment promoting cell adhesion, differentiation and proliferation $(9,10)$.

Chitosan (CS) is a newly developed biomedical material with biocompatibility, low toxicity and biodegradability $(11,12)$, and its polysaccharide side chains contain numerous amino and hydroxyl groups that serve as convenient links for surface modifications. In addition, hydroxyapatite (HA), a common biomaterial with chemical components similar to bone mineral, has been widely used in orthopedics to facilitate bone ingrowth and osseointegration $(13,14)$. The recent appreciation that the requirements of bone tissue engineering scaffolds cannot be fulfilled by any single material, has given rise to the concept of composite bone scaffolds. In this context, CS/HA composites generated by wet chemical methods, such as sol-gel, co-precipitation, in situ compositing and electrochemical deposition (15-17), have been shown to accelerate osseointegration (18).

Since cell differentiation and proliferation can only occur following adhesion of cells to a biocompatible surface (19), facilitation of this process by improving cell-scaffold interactions is a desirable property of a bone scaffold $(20,21)$. To this end, cell-scaffold adhesion has been shown to be promoted by modification of the scaffold using small signaling molecules, such as arginine-glycine-aspartic (RGD) or cell growth 
factors (22), although another study suggested that RGD peptide may actually inhibit bone formation (23). The present study investigated the material-cell interaction characteristics and osseointegrative properties of an RGD-modified CS/HA (RGD-CS/HA) composite scaffold to evaluate its potential application in bone tissue engineering.

\section{Materials and methods}

Isolation and cultivation of bone marrow stromal cells (BMSCs). The present study was approved by the ethics committee of The First Hospital of Harbin City (Harbin, China). Fisher rats $(n=100$; weight range, 100-150 g) were provided by Centre of Experimental Animal of Harbin Medical University (Harbin, China). The animals were maintained in standard lab conditions at $25 \pm 2^{\circ} \mathrm{C}$ in a $12 \mathrm{~h}$ light/dark cycle. The animals were provided with food and water ad libitum. Care of the rats was in compliance with the 'Guide for the Care and Use of Laboratory Animals' published by the National Institute of Health (24). BMSCs were isolated from rat femurs according to the method reported by Tamada and Ikada (25), and cultivated in the control media consisting of complete Dulbecco's modified Eagle's medium (DMEM, Gibco-BRL, Carlsbad, CA, USA) supplemented with $10 \%$ fetal bovine serum (FBS, Gibco-BRL) and $1 \%$ penicillin/streptomycin antibiotics (Mediatech, Inc., Mooresville, NC, USA) in a 5\% $\mathrm{CO}_{2}$ atmosphere at $37^{\circ} \mathrm{C}$. The culture medium was replaced every 2-3 days and cells were passaged at $90 \%$ confluence.

Preparation of the RGD-CS/HA scaffold. The CS/HA composite was prepared by in situ compositing combined with lyophilization. Briefly, $1.25 \mathrm{~g} \mathrm{Ca}\left(\mathrm{NO}_{3}\right)_{2} \bullet 4 \mathrm{H}_{2} \mathrm{O}$ and $0.43 \mathrm{~g} \mathrm{KH}_{2} \mathrm{PO}_{4}$ were added to $100 \mathrm{ml}$ acetic acid, and the solution was stirred until fully dissolved. Then, $5.0 \mathrm{~g}$ CS (Haihui Bioengineering Co., Ltd., Qingdao, China) was added to the solution, and the mixture was stirred for $4 \mathrm{~h}$ at room temperature to obtain a homogeneous $5 \mathrm{wt} \%$ CS solution. Subsequently, the CS solution was cast onto the internal surface of a custom-made mold, and the mold was soaked in $5 \mathrm{wt} \% \mathrm{NaOH}$ to form a CS/HA hydrogel. The hydrogel was washed with deionized water until the $\mathrm{pH}$ value of the water was $\sim 7$, then air-dried in an oven at $60^{\circ} \mathrm{C}$ until a diameter of $10 \mathrm{~mm}$ was reached. The semi-dried CS/HA composite was lyophilized, and cut into CS/HA disks with a diameter of $10 \mathrm{~mm}$ and a height of $1.5 \mathrm{~mm}$. The CS/HA disks were sterilized in an autoclave at $120^{\circ} \mathrm{C}$ for $30 \mathrm{~min}$.

The RGD-CS/HA scaffold was prepared by physical adsorption of the RGD peptide onto the CS/HA disks. Briefly, the sterilized CS/HA samples were immersed in RGD solution (100 mg/l $\mathrm{C}_{12} \mathrm{H}_{22} \mathrm{~N}_{6} \mathrm{O}_{6}$; Sigma-Aldrich, St. Louis, MO, USA) at $37^{\circ} \mathrm{C}$ for $24 \mathrm{~h}$. The RGD-CS/HA scaffold was obtained after washing in phosphate-buffered saline (PBS) three times to remove free reagents, followed by air drying. Spatial distribution of the RGD peptide on the CS/HA samples was determined by labeling the RGD solution with fluorescein isothiocyanate (FITC) followed by visualization using a Nikon E800 fluorescence microscope (Nikon Instruments Inc., Melville, NY, USA).

RGD-CS/HA scaffold-BMSC interactions

Cell adhesion rate. A total of six RGD-CS/HA scaffolds were placed into the well of a 24 -well culture plate. In total,
$1 \times 10^{4}$ cells diluted into $2 \mathrm{ml}$ of control media supplemented with $10^{-9} \mathrm{~mol} / \mathrm{l} \mathrm{Na}-\beta$-glycerophosphate (Trust and Pharmaceutical Co., Ltd., Shanghai, China) and $10^{-8} \mathrm{~mol} / \mathrm{l}$ dexamethasone were then added to each well. The scaffold-cell construct was maintained in cell culture for $4 \mathrm{~h}$ at $37^{\circ} \mathrm{C}$ in a $5 \% \mathrm{CO}_{2}$ atmosphere, after which the RGD-CS/HA scaffolds were washed three times with PBS to remove unattached cells. Adherent cells were removed from the RGD-CS/HA scaffolds using trypsin-EDTA, and counted using a Cell Counting kit-8 (Dojindo Molecular Technologies, Inc., Rockville, MA, USA). The absorbance values were measured at $450 \mathrm{~nm}$ using a Multiskan Mk3 (Thermo Fisher Scientific, Waltham, MA, USA) for the initial cell solution $\left(\mathrm{A}_{\mathrm{i}}\right)$ and the unattached cell solution $\left(\mathrm{A}_{\text {free }}\right)$, and the cell adhesion rate was estimated using the following equation: Cell adhesion rate $=\left(1-\mathrm{A}_{\text {free }} / \mathrm{A}_{\mathrm{i}}\right) \times 100$. The average cell adhesion rate of the six RGD-CS/HA scaffolds was calculated.

Cell viability. BMSC viability on the RGD-CS/HA scaffold was evaluated after 3 days of culture in DMEM supplemented with $10 \%$ FBS using the Live/Dead Viability kit for mammalian cells (Life Technologies, Saint Aubin, France). In this assay, calcein-AM penetrates into the cytosol of viable cells and stains the cells green, while ethidium homodimer-1 stains dead cell nuclei red. Following $30 \mathrm{~min}$ of incubation at room temperature and rinsing with PBS, BMSCs were observed using a FluoVie FV300 confocal laser microscope (Olympus, Tokyo, Japan) and a Nikon E800 fluorescence microscope.

Cell morphology. BMSC morphology on the RGD-CS/HA scaffold was evaluated after 4,24 and 48 h of culture in DMEM supplemented with $10 \%$ FBS. The RGD-CS/HA scaffolds were rinsed thoroughly with PBS to remove the unattached cells, and then fixed using a 2.5\% glutaraldehyde solution (Sigma-Aldrich). Fixed cells were dehydrated progressively in a graded ethanol series, after which RGD-CS/HA scaffolds were sputter-coated with a gold-palladium layer and examined using a scanning electron microscope (SEM, S-3400 N; Hitachi, Toyko, Japan).

Alkaline phosphatase (ALP) activity. Following 14 days of culture under osteogenic conditions (DMEM supplemented with $10 \% \mathrm{FBS}, 50 \mu \mathrm{g} / \mathrm{ml}$ vitamin $\mathrm{C}$ (Trust and Pharmaceutical Co., Ltd.,), $10^{-9} \mathrm{~mol} / \mathrm{l} \mathrm{Na-} \beta$-glycerophosphate and $10^{-8}$ mol/l dexamethasone; Hao Yuan Biological Pharmaceutical Co., Ltd., Shanghai, China), the RGD-CS/HA scaffold was transferred to a $1.5-\mathrm{ml}$ centrifuge tube. The cell membrane was ruptured by ultrasonic cleaning to release the proteins and DNA into the supernatant. Supernatant ALP activity was measured using a rat alkaline phosphatase ELISA kit (R\&D Systems, Minneapolis, MN, USA) according to the manufacturer's instructions. DNA was quantified using a PicoGreen kit (Genmed Scientifics Inc., Wilmington, DE, USA) following standard protocol. The absorbance was read at an excitation/emission of $480-530 \mathrm{~nm}$ on a microplate reader. Three measurements were taken and the results are reported as the mean \pm standard deviation

In vivo heterotopic ossification. The biocompatibility of the RGD-CS/HA scaffold was evaluated through in vivo 

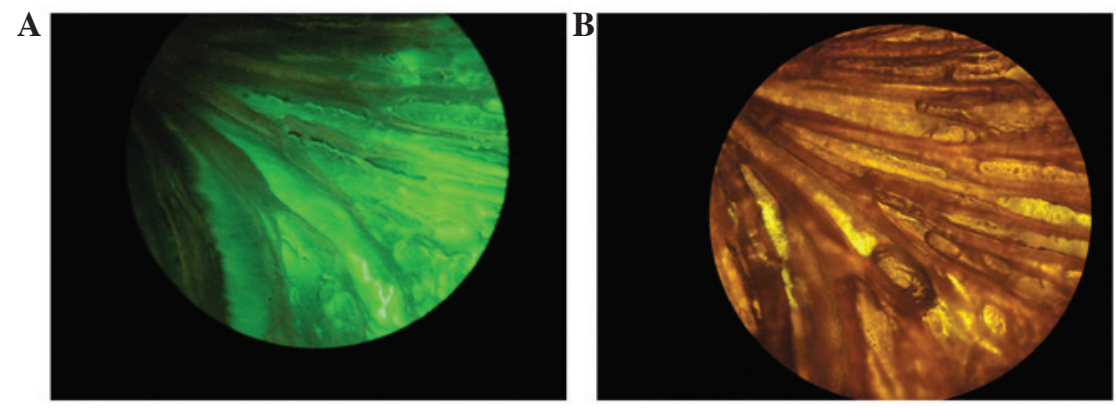

Figure 1. Micrographs of fluorescein isothiocyanate-labeled arginine-glycine-aspartic acid-chitosan/hydroxyapatite scaffold (A) with and (B) without fluorescence mode.
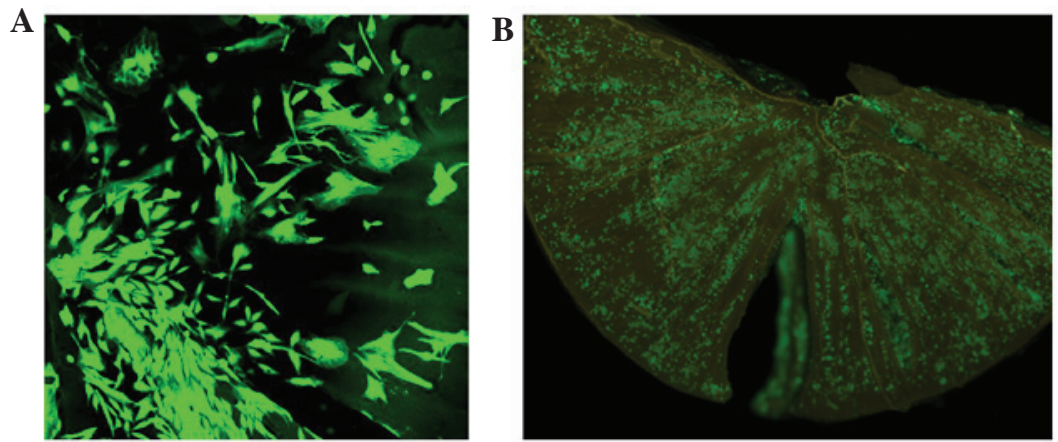

Figure 2. Micrographs of bone marrow stromal cells seeded on the arginine-glycine-aspartic acid-chitosan/hydroxyapatite scaffold after 3 days of culture. (A) Confocal and (B) fluorescence microscopy images.

heterotopic ossification. Male Fisher rats $(\mathrm{n}=60)$ were randomly divided into two groups. Following anesthesia with $10 \%$ chloral hydrate (Trust and Pharmaceutical Co., Ltd.), a $2-\mathrm{cm}$ incision was made in the back, and the subcutaneous tissue and fascia were separated. Subsequently, RGD-CS/HA (RGD-CS/HA group), or CS/HA (CS/HA control group) scaffolds were implanted. The incision was then sutured using Yiqiao absorbable sutures (Johnson \& Johnson, New Brunswick, NJ, USA) and the rat was injected with gentamicin (5 mg/kg; Shanxi Kang Pharmaceutical Co., Ltd., Lihua Yuan, China). After implantation for 1 day, 1 week, 2 weeks, 4 weeks or 6 weeks, respectively (six rats at each time point), scaffolds were collected and examined by X-ray radiography (YSX0101; Yueshen Medical Equipment Co., Ltd., Guangzhou, China). The radiographs were imported using Image Pro Plus software (Media Cybernetics, Inc., Rockville, MD, USA), and the optical density value was calculated. Scaffolds were then fixed using a $10 \%$ glutaraldehyde solution, decalcified, stained with hematoxylin and eosin (H\&E), mounted in resin and sectioned into $5-\mu \mathrm{m}$ samples prior to examination using an optical microscope (S8; Olympus Corporation, Toyko, Japan).

In vivo bone defect repair. The osseointegrative properties of the RGD-CS/HA scaffold were investigated using an in vivo bone defect repair experiment. Briefly, 36 female New Zealand rabbits (weight range, $2.0-2.5 \mathrm{~kg}$ ) provided by the Centre of Experimental Animal of Harbin Medical University were randomly divided into three groups. Rabbits were intravenously anesthetized with $2 \%$ pentobarbital (Wegene Co., Ltd., Shanghai, China), after which a $3-\mathrm{cm}$ incision was made in the radius of the front leg. After separation of subcutaneous tissue and muscle, a bone defect with a length of $1.5 \mathrm{~cm}$ was established, with the osteotomy line $\sim 3 \mathrm{~cm}$ away from the distal end of the radius. For the RGD-CS/HA group, the RGD-CS/HA scaffold was implanted into the bone defect area, and for the CS/HA group, the CS/HA scaffold was used. The incision was then washed with gentamicin and sutured. For the control group, no scaffold was implanted and the incision was sutured directly. The rabbits were injected with $10 \mathrm{mg} / \mathrm{kg}$ penicillin (Shanxi Kang Pharmaceutical Co., Ltd.) for 3 days, then examined by X-ray radiography after implantation for 2, 4 and 8 weeks (four rabbits at each time point). Additionally, for each group, three-dimensional (3D) reconstruction of the bone defect area was performed 8 weeks after implantation of the scaffolds using a electronic computer X-ray tomography technique (Aquilion ONE, Toshiba, Tokyo, Japan; thickness: $0.5 \mathrm{~mm}$ ). Furthermore, the radius of the rabbit in the RGD-CS/HA and CS/HA scaffold groups was obtained 8 weeks after implantation, and the attached ligaments and muscles were carefully dissected. The ends of the radius were mounted with denture powder (Pigeon Dental Mfg. Co., Ltd., Shanghai, China), then placed in an SMD-10 universal material tester (Mechanical Science Co., Ltd., Changchun, China) for three-point bending test with a loading speed of $0.5 \mathrm{~mm} / \mathrm{min}$. The maximum applied load that the radius could bear, and the bending rigidity, were recorded during the experiment, and compared with values obtained for a normal radius. The average value for four rabbits The average value of four rabbits from each group were calculated, and the results were reported as the mean \pm standard deviation. The statistical significance was determined by analysis of variance. $\mathrm{P}<0.05$ was considered to indicate a statistically significant difference. The animals were sacrificed by injecting $4 \%$ pentobarbital sodium 

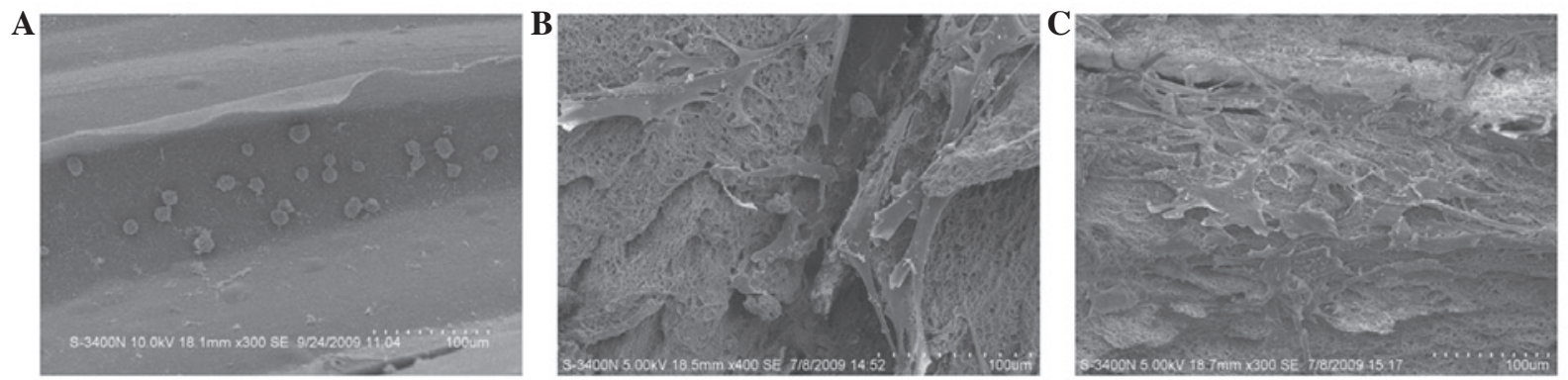

Figure 3. Scanning electron micrographs of bone marrow stromal cells seeded on the arginine-glycine-aspartic acid-chitosan/hydroxyapatite scaffold after (A) 4, (B) 24 and (C) $48 \mathrm{~h}$ incubation.

A
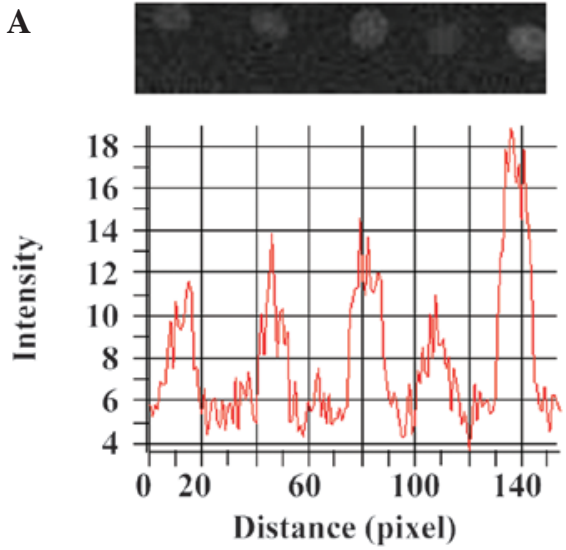

B
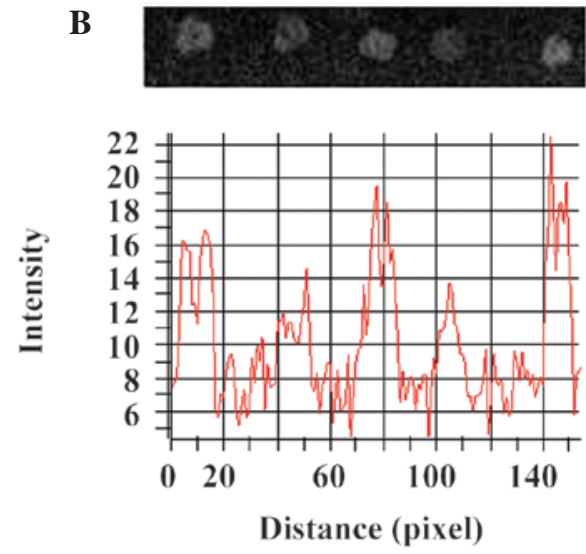

Figure 4. Optical density values of implanted (A) arginine-glycine-aspartic acid-chitosan/hydroxyapatite and (B) chitosan/hydroxyapatite scaffolds after 1 day, and 1,2, 4 and 6 weeks (left to right in images).

A

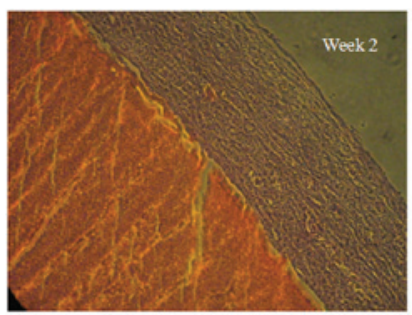

B

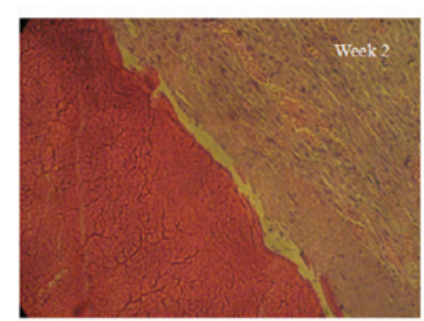

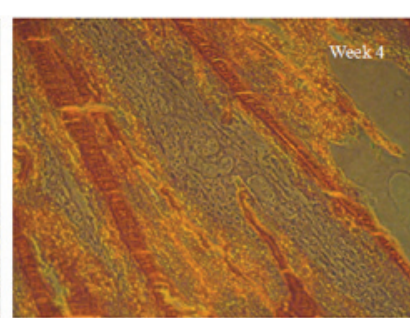

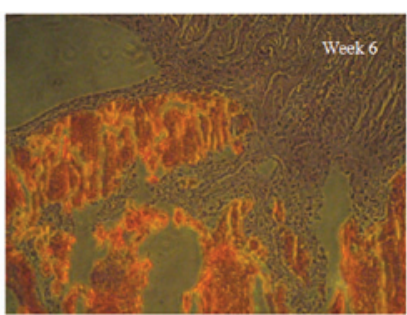

RGD-CS/HA Group
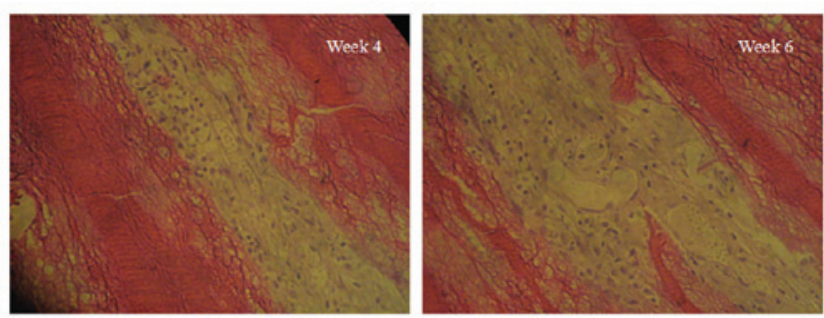

CS/HA Group

Figure 5. Optical micrographs of implanted (A) RGD-CS/HA and (B) CS/HA scaffolds after 2, 4 and 6 weeks. (Hematoxylin and eosin staining; magnification, x20). RGD, arginine-glycine-aspartic acid; CS, chitosan; HA, hydroxyapatite.

(4 ml/kg; Xitang Biological Technology Co., Ltd., Shanghai, China).

Statistical Analysis. The results of the biomechanical assessments are reported as the mean \pm standard deviation and were analyzed using Statistical Product and Service Solutions software (SPSS version 19.0; SPSS, Inc., Chicago, IL, USA). The statistical difference among different groups was evaluated using one-way analysis of variance. $\mathrm{P}<0.05$ was considered to indicate a statistically significant difference. 

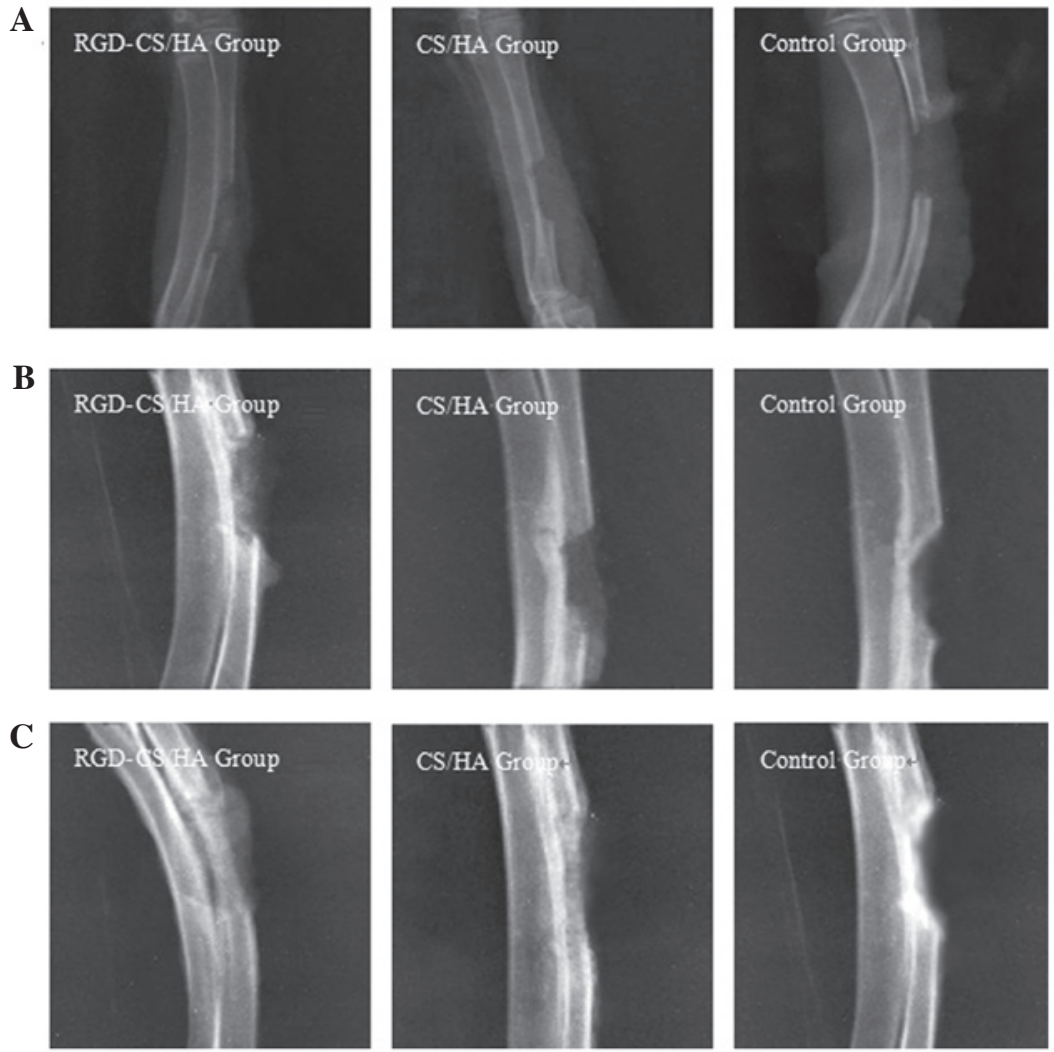

Figure 6. X-ray micrographs of rabbit radial defect repair in the arginine-glycine-aspartic acid-chitosan/hydroxyapatite scaffold, chitosan/hydroxyapatite scaffold and control groups after (A) 2, (B) 4 and (C) 6 weeks.

\section{Results}

Scaffold implantation. Fig. 1 shows micrographs of the FITC-labeled RGD-CS/HA scaffold with and without fluorescence. Under the non-fluorescent mode, the channel morphology of the CS/HA composite was clearly visible, whilst under the fluorescent mode, the CS/HA composite exhibited a homogeneous bright green color, indicating uniform distribution of the RGD peptide.

\section{RGD-CS/HA scaffold-BMSC interactions}

Cell adhesion rate. The adhesion rate of BMSCs to the RGD-CS/HA scaffold in the initial incubation period $(4 \mathrm{~h})$ was $80.7 \%$ when the scaffold was prepared using $100 \mathrm{mg} / \mathrm{l}$ CS solution, compared with $71.6 \%$ for scaffold prepared using $50 \mathrm{mg} / \mathrm{l} \mathrm{CS}$ solution. These results indicate that RGD modification enhances the cell affinity of the CS/HA composite.

Cell viability. Confocal (Fig. 2A) and fluorescence (Fig. 2B) microscopy images of BMSCs in the Live/Dead cell assay show that BMSCs were almost entirely viable, fusiform in shape, and evenly distributed on the RGD-CS/HA scaffold, including the channel-shaped pores. These results indicated the high cytocompatibility between the RGD-CS/HA scaffold and BMSCs.

Cell morphology. SEM images of BMSCs seeded on the RGD-CS/HA scaffold indicate that after $4 \mathrm{~h}$ the BMSCs exhibited a round or elliptical shape with almost no spread on the scaffold (Fig. 3A), whereas after $24 \mathrm{~h}$ of culture, they had a flattened polygonal shape and exhibited pseudopodia (Fig. 3B). After $48 \mathrm{~h}$ of culture (Fig. 3C), BMSCs were well distributed on the scaffold, had formed extracellular matrix, and had made numerous cell-cell interactions.

Alkaline phosphatase (ALP) activity. ALP is an essential enzyme for ossification and is a well-defined marker for cell differentiation to an osteogenic lineage. After 14 days of culture under osteogenic conditions, the ALP content of the BMSCs on the RGD-CS/HA scaffold was elevated $(0.006 \pm 0.0008 \mathrm{U} / \mathrm{l} / \mathrm{ng})$, indicating that the BMSCs on the RGD-CS/HA scaffold differentiated toward an osteogenic phenotype.

In vivo heterotopic ossification. X-ray radiographs and optical density values of the implanted scaffolds in the RGD-CS/HA (Fig. 4A) and CS/HA (Fig. 4B) rat groups after 1 day, and 1, 2, 4 and 6 weeks indicate that, in the two groups, the lowest and highest optical density values were observed 4 and 6 weeks after implantation, respectively.

Optical micrographs. Optical micrographs of the implanted scaffolds in the RGD-CS/HA rat group at 2, 4 and 6 weeks (Fig. 5A), indicate the presence of a small number of chondrocytes and abundant blood vessels in the newly formed tissues 2 weeks after implantation. Osteoclasts and osteoblasts were evident after 4 weeks, whereas 6 weeks after implantation, mature trabecular bone and medullary cavity were observed, with blood vessels in between. By contrast, CS/HA scaffolds were characterized by fibroblast hyperplasia giving rise to predominantly fibrillar connective 

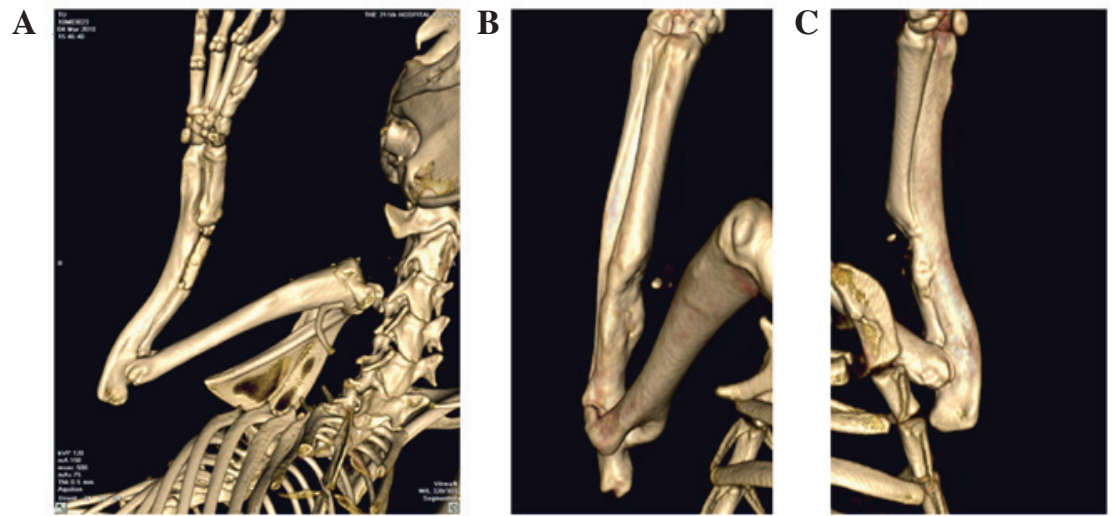

Figure 7.3D reconstruction of rabbit radial defect area after implantation and at 8 weeks post-implantation: (A) 3D recronstruction of rabbit radial defect area following implantation pf the RGD-CS/HA group; (B) RGD-CS/HA group and (C) CS/HA group. Note that the 3D reconstruction images of the RGD-CS/HA and CS/HA groups after implantation are comparable, thus only one image was provided. RGD, arginine-glycine-aspartic acid; CS, chitosan; HA, hydroxyapatite.

Table I. Maximum applied load and bending rigidity of the radius in the different groups.

\begin{tabular}{lcc}
\hline Group & $\begin{array}{c}\text { Maximum applied } \\
\text { load }(\mathrm{N})\end{array}$ & $\begin{array}{c}\text { Bending rigidity } \\
(\mathrm{N} / \mathrm{mm})\end{array}$ \\
\hline Normal radius & $166.22 \pm 18.83^{\mathrm{a}}$ & $62.90 \pm 4.88^{\mathrm{a}}$ \\
RGD-CS/HA & $152.57 \pm 14.63^{\mathrm{a}}$ & $57.80 \pm 5.33^{\mathrm{a}}$ \\
CS/HA & $79.25 \pm 18.35$ & $36.36 \pm 6.35$ \\
\hline
\end{tabular}

${ }^{\text {a }} \mathrm{P}<0.05$, compared with the CS/HA group. RGD, arginine-glycine-aspartic acid; CS, chitosan; HA, hydroxyapatite.

tissues 4 weeks after implantation on the CS/HA scaffold, with only a small amount of trabecular bone and number of collagen fibers visible after 6 weeks. These results indicated the superior biocompatibility of the RGD-CS/HA compared with the CS/HA scaffold.

In vivo bone defect repair. X-ray radiographs comparing rabbit radial bone defects in the RGD-CS/HA, CS/HA and control groups indicate a low density shadow in the RGD-CS/HA group only 2 weeks after implantation (Fig. 6A), whereas 4 weeks after implantation (Fig. 6B), high and low density shadows were observed for the RGD-CS/HA and CS/HA groups, respectively. Eight weeks after implantation (Fig. 6C), de novo bone tissue in the RGD-CS/HA group had a density comparable to that of normal bone, whereas the relatively small diameter of the de novo bone tissue in the CS/HA group indicated that the scaffold had collapsed in vivo. In the control group, a periosteal reaction was observed after 2 and 4 weeks, whereas after 8 weeks an increase in the density of the ends of the radius was observed. 3D images obtained by $\mathrm{X}$-ray tomography (Fig. 7) confirmed that formation of bone tissue after 8 weeks implantation was optimal on the RGD-CS/HA scaffold. Consistent with these data, the maximum applied load and bending rigidity of the radius in the RGD-CS/HA group were comparable with those of the normal group $(\mathrm{P}>0.05)$ and significantly higher than those of the CS/HA group $(\mathrm{P}<0.05$; Table I).

\section{Discussion}

Bone tissue engineering has emerged as an important method in the repair of bone defects, as well as replacement surgery (26-28) and, in this context, there is increasing interest in the development of composite scaffolds with osseointegrative properties. Highly porous scaffolds with open structures are considered to be the optimal candidates for bone substitutes due to their facilitation of bone oxygenation and angiogenesis (29). We have previously shown that a CS/HA composite prepared by in situ compositing in combination with lyophilization contained channel-shaped and spherical pores with average sizes of 400 and $7.8 \mu \mathrm{m}$, respectively, and high porosity (30). The channel-shaped pores provide a framework for bone ingrowth into the pores, and the spherical pores are suitable for capillary ingrowth and extracellular matrix interactions. In the present study, it was demonstrated that an RGD peptide-modified CS/HA composite with increased cellular affinity was superior to the CS/HA composite with respect to cell adhesion rate, cell viability, cell morphology and ALP activity. Furthermore, the scaffold had favorable osseointegrative properties, as measured by in vivo heterotopic ossification and bone defect repair, and possessed biomechanical properties comparable to those of a normal radius. The present results indicate that the RGD-CS/HA scaffold has promising potential for bone tissue engineering applications.

The initial cell adhesion rate on a bone tissue engineering scaffold is crucial for subsequent cell proliferation and differentiation, and exerts a significant influence on the subsequent morphogenesis of artificial tissues. The adhesion rate of BMSCs in the current study is markedly higher than that of rat osteosarcoma cells on RGD-modified CS scaffolds after 1 week of culture (31). Although the difference in the identity of the seed cells may contribute to this discrepancy, it is hypothesized that it is also attributable to the increased access of the BMSCs to the interior of the RGD-CS/HA scaffold afforded by the presence of the channel-shaped pores. Such a notion is supported by our visualization of BMSCs in the channel-shaped pores of the scaffold, as well as by numerous cell-cell interactions and the elevated ALP activity of BMSCs 
on the RGD-CS/HA scaffold, which compares favorably to that of a previous study (32).

The cell affinity of a scaffold is a key consideration in bone tissue engineering. Scaffolds with a 3D porous structure, such as that described, support nutrient supply and promote cell adhesion and proliferation $(33,34)$. The cell adhesion, spreading and cytoskeletal properties of bone scaffolds can be augmented by RGD modification of the scaffold (35), a phenomenon attributed to the formation of focal adhesion between RGD peptide and cell membrane integrins (36). In the present study, the RGD peptide was uniformly immobilized on the CS/HA composite through physical adsorption, a method considered superior to chemical grafting methods employed in other studies $(37,38)$.

The results of the in vivo heterotopic ossification experiments showed that the post-implantation X-ray optical densities of the RGD-CS/HA and CS/HA scaffolds were lowest after 4 weeks and highest after 6 weeks. This may be attributed to the fact that, relative to the rates of osseointegrative indices, rates of CS degradation and $\mathrm{HA}$ adsorption were higher at 4 weeks, and lower at 6 weeks post-implantation. In addition, the superior performance of the RGD-CS/HA scaffold relative to the CS/HA scaffold in the in vivo bone defect repair experiment is attributable to presence of channel-shaped and spherical pores, which stimulate the differentiation of BMSCs into osteoblasts and chondrocytes, and support formation of new bone tissues (39). Furthermore, the elevated $\mathrm{Ca}$ and $\mathrm{P}$ levels that have been reported to accompany HA adsorption may enhance the osseointegrative properties of the differentiated osteoblasts (21).

In conclusion, the present study demonstrated that an RGD-CS/HA scaffold prepared by in situ compositing combined with lyophilization had high biocompatibility, cytocompatibility, histocompatibility and osseointegrative properties, and represents a promising bone substitute for use in bone tissue engineering applications.

\section{Acknowledgements}

The present study was financially supported by Harbin Introduction of Talent Special Fund 2013, Harbin Special Funds for Scientific and Technological Innovation Talent Research (grant no. 2014RFQGJ038). The authors would like to thank Medjaden Bioscience Limited for assisting in the preparation of this manuscript.

\section{References}

1. Langer R and Vacanti JP: Tissue engineering. Science 260: 920-926, 1993

2. Temple HT and Malinin TI: Microparticulate cortical allograft: An alternative to autograft in the treatment of osseous defects. Open Orthop J 2: 91-96, 2008.

3. Zhang HY, Zhang SH, Luo JB, Liu YH, Qian SH, Liang FH and Huang YL: Investigation of protein adsorption mechanism and biotribological properties at simulated stem-cement interface. J Tribology Transactions of ASME 135: 032301, 2013.

4. Zanasi S: Innovations in total knee replacement: New trends in operative treatment and changes in peri-operative management Eur Orthop Traumatol 2: 21-31, 2011.

5. Zhang HY,Luo JB,Zhou M,Zhang Y and Huang YL: Biotribological properties at the stem-cement interface lubricated with different media. J Mech Behav Biomed Mater 20: 209-216, 2013.
6. Miller MA, Ivkovic A, Porter R, Harris MB, Estok DM II, Smith RM, Evans $\mathrm{CH}$ and Vrahas MS: Autologous bone grafting on steroids: Preliminary clinical results. A novel treatment for nonunions and segmental bone defects. Int Orthop 35: 599-605, 2011.

7. Carne GM, Ishaug SL and Mikos AG: Bone tissue engineering. Nat Med 1: 1322-1324, 1995.

8. Baroli B: From natural bone grafts to tissue engineering therapeutics: Brainstorming on pharmaceutical formulative requirements and challenges. J Pharm Sci 98: 1317-1375, 2009.

9. Kamath MS, Ahmed SS, Dhanasekaran M and Santosh SW: Polycaprolactone scaffold engineered for sustained release of resveratrol: Therapeutic enhancement in bone tissue engineering. Int J Nanomedicine 9: 183-195, 2014.

10. Shahini A, Yazdimamaghani M, Walker KJ, Eastman MA, Hatami-Marbini H, Smith BJ, Ricci JL, Madihally SV, Vashaee D and Tayebi L: 3D conductive nanocomposite scaffold for bone tissue engineering. Int J Nanomedicine 9: 167-181, 2014.

11. Saravanan S, Sameera DK, Moorthi A and Selvamurugan N: Chitosan scaffolds containing chicken feather keratin nanoparticles for bone tissue engineering. Int $\mathrm{J}$ Biol Macromol 62: 481-486, 2013.

12. Dessì M, Borzacchiello A, Mohamed TH, Abdel-Fattah WI and Ambrosio L: Novel biomimetic thermosensitive $\beta$-tricalcium phosphate/chitosan-based hydrogels for bone tissue engineering. J Biomed Mater Res A 101: 2984-2993, 2013.

13. Shuai C, Gao C, Feng P, Peng S and Wen X: Grain growth associates mechanical properties in nano-hydroxyapatite bone scaffolds. J Nanosci Nanotechnol 13: 5340-5345, 2013

14. Xia Y, Zhou P, Cheng X, Xie Y, Liang C, Li C and Xu S: Selective laser sintering fabrication of nano-hydroxyapatite/poly- $\varepsilon$-caprolactone scaffolds for bone tissue engineering applications. Int J Nanomedicine 8: 4197-4213, 2013.

15. Yamaguchi I, Tokuchi K, Fukuzaki H, Koyama Y, Takakuda K, Monma $\mathrm{H}$ and Tanaka J: Preparation and microstructure analysis of chitosan/hydroxyapatite nanocomposites. J Biomed Mater Res 55: 20-27, 2001.

16. Zhang Y, Venugopal JR, El-Turki A, Ramakrishna S, Su B and Lim CT: Electrospun biomimetic nanocomposite nanofibers of hydroxyapatite/chitosan for bone tissue engineering. Biomaterials 29: 4314-4322, 2008.

17. Duan K and Wang RZ: Surface modifications of bone implants through wet chemistry. J Mater Chem 16: 2309-2321, 2006.

18. Wang F, Zhang YC, Zhou H, Guo YC and Su XX: Evaluation of in vitro and in vivo osteogenic differentiation of nano-hydroxyapatite/chitosan/poly (lactide-co-glycolide) scaffolds with human umbilical cord mesenchymal stem cells. J Biomed Mater Res A 102: 760-768, 2014.

19. Zhang HY, Han JM, Sun YL, Huang YL and Zhou M: MC3T3-E1 cell response to stainless steel $316 \mathrm{~L}$ with different surface treatments. Mater Sci Eng C Mater Biol Appl 56: 22-29, 2015.

20. El-Ghannam AR, Ducheyne P, Risbud M, Adams CS, Shapiro IM, Castner D, Golledge S and Composto RJ: Model surfaces engineered with nanoscale roughness and RGD tripeptides promote osteoblast activity. J Biomed Mater Res A 68: 615-627, 2004.

21. Secchi AG, Grigoriou V, Shapiro IM, Cavalcanti-Adam EA, Composto RJ, Ducheyne P and Adams CS: RGDS peptides immobilized on titanium alloy stimulate bone cell attachment, differentiation and confer resistance to apoptosis. J Biomed Mater Res A 83: 577-584, 2007.

22. Yang F, Williams CG, Wang DA, Lee H, Manson PN and Elisseeff J: The effect of incorporating RGD adhesive peptide in polyethylene glycol diacrylate hydrogel on osteogenesis of bone marrow stromal cells. Biomaterials 26: 5991-5998, 2005.

23. Sawyer AA, Hennessy KM and Bellis SL: Regulation of mesenchymal stem cell attachment and spreading on hydroxyapatite by RGD peptides and adsorbed serum proteins. Biomaterials 26 : $1467-1475,2005$

24. Clark JD, Gebhart GF, Gonder JC, Keeling ME and Kohn DF. Special Report: The 1996 Guide for the Care and Use of Laboratory Animals. ILAR J 38: 41-48, 1997.

25. Tamada Y and Ikada Y: Fibroblast growth on polymer surfaces and biosynthesis of collagen. J Biomed Mater Res 28: 783-789, 1994.

26. Zhang H, Blunt L, Jiang X, Brown L and Barrans S: The significance of the micropores at the stem-cement interface in total hip replacement. J Biomater Sci Polym Ed 22: 845-856, 2011.

27. Amini AR, Laurencin CT and Nukavarapu SP: Bone tissue engineering: Recent advances and challenges. Crit Rev Biomed Eng 40: 363-408, 2012. 
28. Zhang HY, Blunt LA, Jiang XQ, Brown LT and Barrans SM: The influence of bone cement type on production of fretting wear on the femoral stem surface: A preliminary study. Clin Biomech (Bristol Avon) 27: 666-672, 2012.

29. Roohani SI, Tavangarian F and Emadi R: Nanostructured bioactive glass coating on porous hydroxyapatite scaffold for strength enhancement. Mater Lett 62: 3428-3430, 2008.

30. Qu Z, Yan J, Li B, Zhuang J and Huang Y: Improving bone marrow stromal cell attachment on chitosan/hydroxyapatite scaffolds by an immobilized RGD peptide. Biomed Mater 5: 065001, 2010.

31. Ho MH, Wang DM, Hsieh HJ, Liu HC, Hsien TY, Lai JY and Hou LT: Preparation and characterization of RGD-immobilized chitosan scaffolds. Biomaterials 26: 3197-3206, 2005.

32. Hofmann S, Hagenmuller H, Koch AM, Müller R, Vunjak-Novakovic G, Kaplan DL, Merkle HP and Meinel L: Control of in vitro tissue-engineered bone-like structures using human mesenchymal stem cells and porous silk scaffolds. Biomaterials 28: 1152-1162, 2007.

33. Hutmacher DW: Scaffolds in tissue engineering bone and cartilage. Biomaterials 21: 2529-2543, 2000.

34. Mistry AS, Pham QP, Schouten C, Yeh T, Christenson EM, Mikos AG and Jansen JA: In vivo bone biocompatibility and degradation of porous fumarate-based polymer/alumoxane nanocomposites for bone tissue engineering. J Biomed Mater Res A 92: 451-462, 2010.
35. El-Ghannam AR, Ducheyne P, Risbud M, Adams CS, Shapiro IM, Castner D, Golledge S and Composto RJ: Model surfaces engineered with nanoscale roughness and RGD tripeptides promote osteoblast activity. J Biomed Mater Res A 68: 615-627, 2004.

36. LeBaron RG and Athanasiou KA: Extracellular matrix cell adhesion peptides: Functional applications in orthopedic materials. Tissue Eng 6: 85-103, 2000.

37. Kantlehner M, Finsinger D, Meyer J, Schaffner P, Jonczyk A, Diefenbach B, Nies B and Kessler H: Selective RGD-mediated adhesion of osteoblasts at surfaces of implants. Angew Chem Int Ed 38: 560-562, 1999 .

38. Haubner R, Bruchertseifer F, Bock M, Kessler H, Schwaiger M and Wester HJ: Synthesis and biological evaluation of a (99m) Tc-labelled cyclic RGD peptide for imaging the alphavbeta3 expression. Nuklearmedizin 43: 26-32, 2004.

39. Oliveira JM, Rodrigues MT, Silva SS, Malafaya PB, Gomes ME, Viegas CA, Dias IR, Azevedo JT, Mano JF and Reis RL: Novel hydroxyapatite/chitosan bilayered scaffold for osteochondral tissue-engineering applications: Scaffold design and its performance when seeded with goat bone marrow stromal cells. Biomaterials 27: 6123-6137, 2006. 